\title{
Tempered Radon Measures
}

\author{
Maryia KaBANAVA
}

\author{
Mathematical Institute \\ Friedrich Schiller University Jena \\ D-07737 Jena, Germany \\ kabanova@minet.uni-jena.de
}

Received: December 11, 2007

Accepted: January 1, 2008

\begin{abstract}
A tempered Radon measure is a $\sigma$-finite Radon measure in $\mathbb{R}^{n}$ which generates a tempered distribution. We prove the following assertions. A Radon measure $\mu$ is tempered if, and only if, there is a real number $\beta$ such that $\left(1+|x|^{2}\right)^{\frac{\beta}{2}} \mu$ is finite. A Radon measure is finite if, and only if, it belongs to the positive cone $\stackrel{+}{B}_{1 \infty}^{0}\left(\mathbb{R}^{n}\right)$ of $B_{1 \infty}^{0}\left(\mathbb{R}^{n}\right)$. Then $\mu\left(\mathbb{R}^{n}\right) \sim\left\|\mu \mid B_{1 \infty}^{0}\left(\mathbb{R}^{n}\right)\right\|$ (equivalent norms).
\end{abstract}

Key words: Radon measure, tempered distributions, Besov spaces 2000 Mathematics Subject Classification: 42B35, 28C05.

\section{Introduction}

A substantial part of fractal geometry and fractal analysis deals with Radon measures in $\mathbb{R}^{n}$ (also called fractal measures) with compact support. One may consult [5] and the references given there. In the present paper we clarify the relation between arbitrary $\sigma$-finite Radon measure in $\mathbb{R}^{n}$, tempered distributions and weighted Besov spaces. It comes out that a $\sigma$-finite Radon measure $\mu$ in $\mathbb{R}^{n}$ can be identified with a tempered distribution $\mu \in S^{\prime}\left(\mathbb{R}^{n}\right)$ if and only if there is a real number $\beta$ such that

$$
\mu_{\beta}\left(\mathbb{R}^{n}\right)<\infty, \quad \text { where } \quad \mu_{\beta}=\left(1+|x|^{2}\right)^{\frac{\beta}{2}} \mu .
$$

Radon measures $\mu$ with $\mu\left(\mathbb{R}^{n}\right)<\infty$ are called finite. These finite Radon measures can be identified with the positive cone $\stackrel{+}{B}_{1 \infty}^{0}\left(\mathbb{R}^{n}\right)$ of the distinguished Besov space $B_{1 \infty}^{0}\left(\mathbb{R}^{n}\right)$ and

$$
\left\|\mu \mid B_{1 \infty}^{0}\left(\mathbb{R}^{n}\right)\right\| \sim \mu\left(\mathbb{R}^{n}\right)
$$


(equivalent norms).

This paper is organised as follows. In section 1 we collect the definitions and preliminaries. We introduce the well-known weighted Besov spaces $B_{p q}^{s}\left(\mathbb{R}^{n},\langle x\rangle^{\alpha}\right)$ and prove that for fixed $p, q$ with $0<p, q \leq \infty$

and

$$
S\left(\mathbb{R}^{n}\right)=\bigcap_{\alpha, s \in \mathbb{R}} B_{p q}^{s}\left(\mathbb{R}^{n},\langle x\rangle^{\alpha}\right)
$$

$$
S^{\prime}\left(\mathbb{R}^{n}\right)=\bigcup_{\alpha, s \in \mathbb{R}} B_{p q}^{s}\left(\mathbb{R}^{n},\langle x\rangle^{\alpha}\right) .
$$

Although known to specialists we could not find an explicit reference. In section 2 we prove in the Theorems 2.1 and 2.2 the above indicated main results.

\section{Definitions and preliminaries}

Let $\mathbb{N}$ be the collection of all natural numbers and $\mathbb{N}_{0}=\mathbb{N} \cup\{0\}$. Let $\mathbb{R}^{n}$ be Euclidean $n$-space, where $n \in \mathbb{N}$. Put $\mathbb{R}=\mathbb{R}^{1}$, whereas $\mathbb{C}$ is the complex plane. Let $S\left(\mathbb{R}^{n}\right)$ be the Schwartz space of all complex-valued, rapidly decreasing, infinitely differentiable functions on $\mathbb{R}^{n}$. By $S^{\prime}\left(\mathbb{R}^{n}\right)$ we denote its topological dual, the space of all tempered distributions on $\mathbb{R}^{n}$. $L_{p}\left(\mathbb{R}^{n}\right)$ with $0<p \leq \infty$, is the standard quasi-Banach space with respect to Lebesgue measure, quasi-normed by

$$
\left\|f \mid L_{p}\left(\mathbb{R}^{n}\right)\right\|=\left(\int_{\mathbb{R}^{n}}|f(x)|^{p} d x\right)^{\frac{1}{p}}, \quad 0<p<\infty
$$

with the standard modification if $p=\infty$.

If $\varphi \in S\left(\mathbb{R}^{n}\right)$ then

$$
\hat{\varphi}(\xi)=F \varphi(\xi)=(2 \pi)^{-\frac{n}{2}} \int_{\mathbb{R}^{n}} \varphi(x) e^{-i x \xi} d x, \quad \xi \in \mathbb{R}^{n},
$$

denotes the Fourier transform of $\varphi$. The inverse Fourier transform is given by

$$
\check{\varphi}(x)=F^{-1} \varphi(x)=(2 \pi)^{-\frac{n}{2}} \int_{\mathbb{R}^{n}} \varphi(\xi) e^{i x \xi} d \xi, \quad x \in \mathbb{R}^{n} .
$$

One extends $F$ and $F^{-1}$ in the usual way from $S$ to $S^{\prime}$. For $f \in S^{\prime}\left(\mathbb{R}^{n}\right)$,

$$
F f(\varphi)=f(F \varphi), \quad \varphi \in S\left(\mathbb{R}^{n}\right) .
$$

Let $\varphi_{0} \in S\left(\mathbb{R}^{n}\right)$ with

$$
\varphi_{0}(x)=1, \quad|x| \leq 1 \quad \text { and } \quad \varphi_{0}(x)=0, \quad|x| \geq \frac{3}{2},
$$


and let

$$
\varphi_{k}(x)=\varphi_{0}\left(2^{-k} x\right)-\varphi_{0}\left(2^{-k+1} x\right), \quad x \in \mathbb{R}^{n}, \quad k \in \mathbb{N} .
$$

Then, since

$$
1=\sum_{j=0}^{\infty} \varphi_{j}(x) \text { for all } x \in \mathbb{R}^{n},
$$

the $\varphi_{j}$ form a dyadic resolution of unity in $\mathbb{R}^{n} \cdot\left(\varphi_{k} \hat{f}\right)^{\sim}$ is an entire analytic function on $\mathbb{R}^{n}$ for any $f \in S^{\prime}\left(\mathbb{R}^{n}\right)$. In particular, $\left(\varphi_{k} \hat{f}\right)^{\swarrow}(x)$ makes sense pointwise.

Definition 1.1. Let $\varphi=\left\{\varphi_{j}\right\}_{j=0}^{\infty}$ be the dyadic resolution of unity according to (1)-(3), $s \in \mathbb{R}, 0<p \leq \infty, 0<q \leq \infty$, and

$$
\left\|f \mid B_{p q}^{s}\left(\mathbb{R}^{n}\right)\right\|_{\varphi}=\left(\sum_{j=0}^{\infty} 2^{j s q}\left\|\left(\varphi_{k} \hat{f}\right)^{\sim} \mid L_{p}\left(\mathbb{R}^{n}\right)\right\|^{q}\right)^{\frac{1}{q}}
$$

(with the usual modification if $q=\infty$ ). Then the Besov space $B_{p q}^{s}\left(\mathbb{R}^{n}\right)$ consists of all $f \in S^{\prime}\left(\mathbb{R}^{n}\right)$ such that $\left\|f \mid B_{p q}^{s}\left(\mathbb{R}^{n}\right)\right\|_{\varphi}<\infty$.

We denote by $L_{p}\left(\mathbb{R}^{n},\langle x\rangle^{\alpha}\right)$, where

$$
\langle x\rangle^{\alpha}=\left(1+|x|^{2}\right)^{\frac{\alpha}{2}},
$$

the weighted $L_{p}$-space quasi-normed by

$$
\left\|f\left|L_{p}\left(\mathbb{R}^{n},\langle x\rangle^{\alpha}\right)\|=\|\langle\cdot\rangle^{\alpha} f\right| L_{p}\left(\mathbb{R}^{n}\right)\right\| .
$$

Definition 1.2. Let $\varphi=\left\{\varphi_{j}\right\}_{j=0}^{\infty}$ be the dyadic resolution of unity according to (1)-(3), $s \in \mathbb{R}, 0<p \leq \infty, 0<q \leq \infty$. Then the weighted Besov space $B_{p q}^{s}\left(\mathbb{R}^{n},\langle x\rangle^{\alpha}\right)$ is a collection of all $f \in S^{\prime}\left(\mathbb{R}^{n}\right)$ such that

$$
\left\|f \mid B_{p q}^{s}\left(\mathbb{R}^{n},\langle x\rangle^{\alpha}\right)\right\|_{\varphi}=\left(\sum_{j=0}^{\infty} 2^{j s q}\left\|\left(\varphi_{k} \hat{f}\right)^{\sim} \mid L_{p}\left(\mathbb{R}^{n},\langle x\rangle^{\alpha}\right)\right\|^{q}\right)^{\frac{1}{q}}
$$

(with the usual modification if $q=\infty$ ) is finite.

Remark 1.3. If $\alpha=0$ then we have the space $B_{p q}^{s}\left(\mathbb{R}^{n}\right)$ as introduced in Definition 1.1. It is also known from $\left[1\right.$, ch. 4.2.2] that the operator $f \mapsto\langle x\rangle^{\alpha} f$ is an isomorphic mapping from $B_{p q}^{s}\left(\mathbb{R}^{n},\langle x\rangle^{\alpha}\right)$ onto $B_{p q}^{s}\left(\mathbb{R}^{n}\right)$. In particular,

$$
\left\|\langle\cdot\rangle^{\alpha} f\left|B_{p q}^{s}\left(\mathbb{R}^{n}\right)\|\sim\| f\right| B_{p q}^{s}\left(\mathbb{R}^{n},\langle x\rangle^{\alpha}\right)\right\| .
$$

Next we review some special properties of weighted Besov spaces. 
Proposition 1.4. For fixed $0<p, q \leq \infty$

$$
S\left(\mathbb{R}^{n}\right)=\bigcap_{\alpha, s \in \mathbb{R}} B_{p q}^{s}\left(\mathbb{R}^{n},\langle x\rangle^{\alpha}\right)
$$

and

$$
S^{\prime}\left(\mathbb{R}^{n}\right)=\bigcup_{\alpha, s \in \mathbb{R}} B_{p q}^{s}\left(\mathbb{R}^{n},\langle x\rangle^{\alpha}\right) .
$$

Proof. Step 1. The inclusion

is clear.

$$
S\left(\mathbb{R}^{n}\right) \subset \bigcap_{\alpha, s \in \mathbb{R}} B_{p q}^{s}\left(\mathbb{R}^{n},\langle x\rangle^{\alpha}\right)
$$

To prove that any $f \in \bigcap_{\alpha, s \in \mathbb{R}} B_{p q}^{s}\left(\mathbb{R}^{n},\langle x\rangle^{\alpha}\right)$ belongs to $S\left(\mathbb{R}^{n}\right)$, it is sufficient to show that for any fixed $N \in \mathbb{N}$ there are $\alpha(N) \in \mathbb{R}$ and $s(N) \in \mathbb{R}$ such that

$$
\sup _{|\beta| \leq N} \sup _{x \in \mathbb{R}^{n}}\langle x\rangle^{2 N}\left|D^{\beta} f(x)\right| \leq c\left\|f \mid B_{p q}^{s}\left(\mathbb{R}^{n},\langle x\rangle^{\alpha}\right)\right\| .
$$

For any multiindex $\beta$ there are polynomials $P_{\gamma}^{\beta}, \operatorname{deg} P_{\gamma}^{\beta} \leq 2 N$ such that

Hence

$$
\langle x\rangle^{2 N} D^{\beta} f(x)=\sum_{\gamma \leq \beta} D^{\gamma}\left[\left(P_{\gamma}^{\beta} f\right)(x)\right] .
$$

$$
\begin{aligned}
\sup _{|\beta| \leq N} \sup _{x \in \mathbb{R}^{n}}\langle x\rangle^{2 N}\left|D^{\beta} f(x)\right| & =\sup _{|\beta| \leq N} \sup _{x \in \mathbb{R}^{n}}\left|\sum_{\gamma \leq \beta} D^{\gamma}\left[\left(P_{\gamma}^{\beta} f\right)(x)\right]\right| \\
& \leq \sup _{|\beta| \leq N} \sum_{|\gamma| \leq N} \sup _{x \in \mathbb{R}^{n}}\left|D^{\gamma}\left[\left(P_{\gamma}^{\beta} f\right)(x)\right]\right| \\
& \leq \sup _{|\beta| \leq N} \sum_{|\gamma| \leq N}\left\|P_{\gamma}^{\beta} f \mid C^{N}\left(\mathbb{R}^{n}\right)\right\| .
\end{aligned}
$$

Due to the embedding theorems [3, ch. 2.7.1],

$$
\begin{aligned}
\left\|P_{\gamma}^{\beta} f \mid C^{N}\left(\mathbb{R}^{n}\right)\right\| & \leq c\left\|P_{\gamma}^{\beta} f \mid B_{p q}^{N+\frac{n}{p}+\varepsilon}\left(\mathbb{R}^{n}\right)\right\| \\
& =c\left\|\frac{P_{\gamma}^{\beta}}{\langle x\rangle^{2 N}}\langle x\rangle^{2 N} f \mid B_{p q}^{N+\frac{n}{p}+\varepsilon}\left(\mathbb{R}^{n}\right)\right\|
\end{aligned}
$$

for any $\varepsilon>0 . \frac{P_{\gamma}^{\beta}}{\langle x\rangle^{2 N}}$ is a pointwise multiplier for $B_{p q}^{N+\frac{n}{p}+\varepsilon}\left(\mathbb{R}^{n}\right)[3$, ch. 2.8.2]. Therefore

$$
\begin{aligned}
\| \frac{P_{\gamma}^{\beta}}{\langle x\rangle^{2 N}}\langle x\rangle^{2 N} f \mid & B_{p q}^{N+\frac{n}{p}+\varepsilon}\left(\mathbb{R}^{n}\right) \| \\
& \leq c\left\|\frac{P_{\gamma}^{\beta}}{\langle x\rangle^{2 N}}\left|\mathcal{C}^{N+\frac{n}{p}+\varepsilon}\left(\mathbb{R}^{n}\right)\|\cdot\|\langle x\rangle^{2 N} f\right| B_{p q}^{N+\frac{n}{p}+\varepsilon}\left(\mathbb{R}^{n}\right)\right\| .
\end{aligned}
$$


According to Remark 1.3

$$
\left\|\langle x\rangle^{2 N} f\left|B_{p q}^{N+\frac{n}{p}+\varepsilon}\left(\mathbb{R}^{n}\right)\|\sim\| f\right| B_{p q}^{N+\frac{n}{p}+\varepsilon}\left(\mathbb{R}^{n},\langle x\rangle^{2 N}\right)\right\| .
$$

Combining (5)-(8), one gets

$$
\begin{aligned}
\sup _{|\beta| \leq N} \sup _{x \in \mathbb{R}^{n}}\langle x\rangle^{2 N}\left|D^{\beta} f(x)\right| & \leq c \sum_{|\gamma| \leq N}\left\|\langle x\rangle^{2 N} f \mid B_{p q}^{N+\frac{n}{p}+\varepsilon}\left(\mathbb{R}^{n}\right)\right\| \\
& \leq c\left\|f \mid B_{p q}^{N+\frac{n}{p}+\varepsilon}\left(\mathbb{R}^{n},\langle x\rangle^{2 N}\right)\right\|
\end{aligned}
$$

and it follows (4).

Step 2. Let $1<p \leq \infty, 1<q \leq \infty$ and let $p^{\prime}$ and $q^{\prime}$ be defined in the standard way by

$$
\frac{1}{p}+\frac{1}{p^{\prime}}=1, \quad \frac{1}{q}+\frac{1}{q^{\prime}}=1 .
$$

The inclusion

$$
\bigcup_{\alpha, s \in \mathbb{R}} B_{p q}^{s}\left(\mathbb{R}^{n},\langle x\rangle^{\alpha}\right) \subset S^{\prime}\left(\mathbb{R}^{n}\right)
$$

is evident.

As far as the opposite inclusion is concerned, we recall that $f \in S^{\prime}\left(\mathbb{R}^{n}\right)$ if and only if there are $l \in \mathbb{N}$ and $m \in \mathbb{N}$ such that

$$
|f(\varphi)| \leq c \sup _{|\alpha| \leq m} \sup _{x \in \mathbb{R}^{n}}\langle x\rangle^{l}\left|D^{\alpha} \varphi(x)\right|,
$$

for all $\varphi \in S\left(\mathbb{R}^{n}\right)$. By $(9)$,

$$
\sup _{|\alpha| \leq m} \sup _{x \in \mathbb{R}^{n}}\langle x\rangle^{l}\left|D^{\alpha} \varphi(x)\right| \leq c\left\|\varphi \mid B_{p^{\prime} q^{\prime}}^{m+\frac{n}{p}+\varepsilon}\left(\mathbb{R}^{n},\langle x\rangle^{l}\right)\right\| .
$$

According to our choice of $p$ and $q$, it follows that $1 \leq p^{\prime}<\infty$ and $1 \leq q^{\prime}<\infty$. Thus, by $[3$, ch. 2.11.2],

$$
f \in\left(B_{p^{\prime} q^{\prime}}^{m+\frac{n}{p}+\varepsilon}\left(\mathbb{R}^{n},\langle x\rangle^{l}\right)\right)^{\prime}=B_{p q}^{-\left(m+\frac{n}{p}+\varepsilon\right)}\left(\mathbb{R}^{n},\langle x\rangle^{-l}\right) .
$$

This means

$$
S^{\prime}\left(\mathbb{R}^{n}\right) \subset \bigcup_{\alpha, s \in \mathbb{R}} B_{p q}^{s}\left(\mathbb{R}^{n},\langle x\rangle^{\alpha}\right) .
$$

Step 3. Let $0<p \leq 1,1<q \leq \infty$. By the arguments above, for $f \in S^{\prime}\left(\mathbb{R}^{n}\right)$ there are $\alpha \in \mathbb{R}$ and $s \in \mathbb{R}$ such that

$$
f \in B_{\infty q}^{s}\left(\mathbb{R}^{n},\langle x\rangle^{\alpha}\right) .
$$


We want to show that

$$
f \in B_{p q}^{s}\left(\mathbb{R}^{n},\langle x\rangle^{\alpha-\gamma}\right), \quad \gamma>\frac{n}{p}
$$

Indeed,

$$
\begin{aligned}
\left\|f \mid B_{p q}^{s}\left(\mathbb{R}^{n},\langle x\rangle^{\alpha-\gamma}\right)\right\| & =\left(\sum_{j=0}^{\infty} 2^{j s q}\left\|\langle x\rangle^{\alpha-\gamma}\left(\varphi_{j} \hat{f}\right)^{\curlywedge} \mid L_{p}\left(\mathbb{R}^{n}\right)\right\|^{q}\right)^{\frac{1}{q}} \\
& \leq\left(\sum_{j=0}^{\infty} 2^{j s q} \sup _{x \in \mathbb{R}^{n}}\left[\langle x\rangle^{\alpha}\left|\left(\varphi_{j} \hat{f}\right)^{\swarrow}(x)\right|\right]^{q}\left(\int_{\mathbb{R}^{n}}\langle x\rangle^{-\gamma p} d x\right)^{\frac{q}{p}}\right)^{\frac{1}{q}} \\
& \leq c\left\|f \mid B_{\infty q}^{s}\left(\mathbb{R}^{n},\langle x\rangle^{\alpha}\right)\right\| .
\end{aligned}
$$

Step 4. When $0<q \leq 1$, first we may find $\alpha \in \mathbb{R}$ and $s \in \mathbb{R}$ such that

$$
f \in B_{p q^{*}}^{s}\left(\mathbb{R}^{n},\langle x\rangle^{\alpha}\right),
$$

$q^{*}>1$, and then use the fact that

$$
B_{p q *}^{s}\left(\mathbb{R}^{n},\langle x\rangle^{\alpha}\right) \subset B_{p q}^{s-\varepsilon}\left(\mathbb{R}^{n},\langle x\rangle^{\alpha}\right), \quad \varepsilon>0 .
$$

Next we recall some notation. A measure $\mu$ is called $\sigma$-finite in $\mathbb{R}^{n}$ if for any $R>0$,

$$
\mu(\{x:|x|<R\})<\infty .
$$

A measure $\mu$ is a Radon measure if all Borel sets are $\mu$ measurable and

(i) $\mu(K)<\infty$ for compact sets $K \subset \mathbb{R}^{n}$,

(ii) $\mu(V)=\sup \{\mu(K): K \subset V$ is compact $\}$ for open sets $V \subset \mathbb{R}^{n}$,

(iii) $\mu(A)=\inf \{\mu(V): A \subset V, V$ is open $\}$ for $A \subset \mathbb{R}^{n}$.

Let $\mu$ be a positive Radon measure in $\mathbb{R}^{n}$. Let $T_{\mu}$,

$$
T_{\mu}: \varphi \longmapsto \int_{\mathbb{R}^{n}} \varphi(x) \mu(d x), \quad \varphi \in S\left(\mathbb{R}^{n}\right),
$$

be the linear functional generated by $\mu$.

Definition 1.5. A positive Radon measure $\mu$ is said to be tempered if $T_{\mu} \in S^{\prime}\left(\mathbb{R}^{n}\right)$.

Proposition 1.6. Let $\mu^{1}$ and $\mu^{2}$ be two tempered Radon measures. Then

$$
T_{\mu^{1}}=T_{\mu^{2}} \text { in } S^{\prime}\left(\mathbb{R}^{n}\right) \quad \text { if, and only if, } \mu^{1}=\mu^{2} .
$$

Proof. The Proposition is valid by the arguments in [5, p. 80]. 
This justifies the identification of $\mu$ and correspondent tempered distribution $T_{\mu}$ and we may write $\mu \in S^{\prime}\left(\mathbb{R}^{n}\right)$.

Definition 1.7. $f \in S^{\prime}\left(\mathbb{R}^{n}\right)$ is called a positive distribution if

$$
f(\varphi) \geq 0 \quad \text { for any } \varphi \in S\left(\mathbb{R}^{n}\right) \text { with } \varphi \geq 0 .
$$

If $f \in L_{1}^{\text {loc }}\left(\mathbb{R}^{n}\right)$ then $f \geq 0$ means $f(x) \geq 0$ almost everywhere.

Remark 1.8. If $f$ is a positive distribution, then $f \in C_{0}\left(\mathbb{R}^{n}\right)^{\prime}$ and it follows from the Radon-Riesz theorem that there is a tempered Radon measure $\mu$ such that

$$
f(\varphi)=\int_{\mathbb{R}^{n}} \varphi(x) \mu(d x)
$$

$[2$, pp. $61,62,71,75]$.

\section{Main assertions}

Our next result refers to tempered measures.

\section{Theorem 2.1.}

(i) A Radon measure $\mu$ in $\mathbb{R}^{n}$ is tempered if, and only if, there is a real number $\beta$ such that $\langle x\rangle^{\beta} \mu$ is finite.

(ii) Let $\mu$ be a tempered Radon measure in $\mathbb{R}^{n}$. Let $j \in \mathbb{N}$,

$$
A_{j}=\left\{x: 2^{j-1} \leq|x| \leq 2^{j+1}\right\}, \quad A_{0}=\{x:|x| \leq 2\} .
$$

Then for some $c>0, \alpha \geq 0$,

$$
\mu\left(A_{k}\right) \leq c 2^{k \alpha} \quad \text { for all } k \in \mathbb{N}_{0} .
$$

Proof. Step 1. First we prove part (ii). Suppose that the assertion does not hold. Then for $c=1$ and $l \in \mathbb{N}$ there is $k_{l} \in \mathbb{N}_{0}$ such that

$$
\mu\left(A_{k_{l}}\right)>2^{k_{l} l} .
$$

As soon as it is found one $k_{l}$ with (10), it follows that there are infinitely many $k_{l}^{m}$, $m \in \mathbb{N}$, that satisfy (10).

With $j \in \mathbb{N}$,

$$
A_{j}^{*}=\left\{x: 2^{j-2} \leq|x| \leq 2^{j+2}\right\}, \quad A_{0}^{*}=\{x:|x| \leq 4\} .
$$

For $l=1$ take any of $k_{1}^{m}$, let it be $k_{1}$. For $l=2$ choose $k_{2} \gg k_{1}$ in such a way that $A_{k_{1}}^{*}$ and $A_{k_{2}}^{*}$ have an empty intersection. For arbitrary $l \in \mathbb{N}$ take

$$
k_{l} \gg k_{l-1} \quad \text { and } \quad A_{k_{l-1}}^{*} \cap A_{k_{l}}^{*}=\emptyset .
$$


Let $\varphi_{0}$ be a $C^{\infty}$ function on $\mathbb{R}^{n}$ with

$$
\varphi_{0}(x)=1, \quad|x| \leq 2 \quad \text { and } \quad \varphi_{0}(x)=0, \quad|x| \geq 4
$$

Let $k \in \mathbb{N}$ and

$$
\varphi_{k}(x)=\varphi_{0}\left(2^{-k} x\right)-\varphi_{0}\left(2^{-k+3} x\right), \quad x \in \mathbb{R}^{n} .
$$

Then we have

$$
\operatorname{supp} \varphi_{k} \subset A_{k}^{*}
$$

and

$$
\varphi_{k}(x)=1, \quad x \in A_{k} .
$$

Let

$$
\varphi(x)=\sum_{l=1}^{\infty} 2^{-l k_{l}} \varphi_{k_{l}}(x) .
$$

For any fixed $N \in \mathbb{N}_{0}$

$$
\begin{aligned}
\sup _{|\alpha| \leq N} \sup _{x \in \mathbb{R}^{n}}\left(1+|x|^{2}\right)^{N}\left|D^{\alpha} \varphi(x)\right| & \\
& =\sup _{|\alpha| \leq N} \sup _{x \in \mathbb{R}^{n}}\left(1+|x|^{2}\right)^{N}\left|D^{\alpha}\left(\sum_{l=1}^{\infty} 2^{-l k_{l}} \varphi_{k_{l}}(x)\right)\right| \\
& \leq \sup _{l \in \mathbb{N}} \sup _{|\alpha| \leq N} \sup _{x \in \mathbb{R}^{n}} 2^{-l k_{l}} 2^{-|\alpha| k_{l}} 2^{|\alpha|}\left(1+|x|^{2}\right)^{N}\left|\left(D^{\alpha} \varphi_{1}\right)\left(2^{-k_{l}+1} x\right)\right| .
\end{aligned}
$$

The last inequality holds, since the functions $\varphi_{k_{l}}$ have disjoint supports. With the change of variables

$$
x^{\prime}=2^{-k_{l}+1} x
$$

one gets

$$
\begin{aligned}
\sup _{|\alpha| \leq N} \sup _{x \in \mathbb{R}^{n}}\left(1+|x|^{2}\right)^{N}\left|D^{\alpha} \varphi(x)\right| & \\
& \leq \sup _{l \in \mathbb{N}} \sup _{|\alpha| \leq N} 2^{-l k_{l}} 2^{-|\alpha| k_{l}} 2^{|\alpha|} 2^{2\left(k_{l}-1\right) N} \sup _{x \in \mathbb{R}^{n}}\left(1+|x|^{2}\right)^{N}\left|D^{\alpha} \varphi_{1}(x)\right| \\
& \leq c \sup _{l \in \mathbb{N}} \sup _{|\alpha| \leq N} 2^{-k_{l}(l+|\alpha|-2 N)} \leq c \sup _{l \in \mathbb{N}} 2^{-k_{l}(l-2 N)} .
\end{aligned}
$$

Since $N$ is fixed and $l$ is tending to infinity, $2^{-k_{l}(l-2 N)}$ is bounded. Thus $\varphi \in S\left(\mathbb{R}^{n}\right)$.

According to the definition of tempered Radon measures

$$
\int_{\mathbb{R}^{n}} \psi(x) \mu(d x)<+\infty
$$


for any $\psi \in S\left(\mathbb{R}^{n}\right)$, but

$$
\int_{\mathbb{R}^{n}} \varphi(x) \mu(d x) \geq \sum_{l=1}^{\infty} \int_{A_{k_{l}}} \varphi(x) \mu(d x) \geq \sum_{l=1}^{\infty} 2^{-l k_{l}} 2^{l k_{l}}=+\infty .
$$

This means that our assertion (10) is false.

Step 2. We prove part (i). Since $\langle x\rangle^{\beta} \mu$ is finite, it is tempered. Then $\mu$ is also tempered. To prove the other direction we take $\beta=-(\alpha+1)$. Then we get

$$
\begin{aligned}
\langle\cdot\rangle^{\beta} \mu\left(\mathbb{R}^{n}\right) & =\int_{\mathbb{R}^{n}}\langle x\rangle^{-(\alpha+1)} \mu(d x) \leq \sum_{k=0}^{\infty} \int_{A_{k}}\langle x\rangle^{-(\alpha+1)} \mu(d x) \\
& \leq c \sum_{k=0}^{\infty} 2^{-k(\alpha+1)} \int_{A_{k}} \mu(d x) \leq c \sum_{k=0}^{\infty} 2^{-k(\alpha+1)} 2^{k \alpha}<\infty .
\end{aligned}
$$

In order to characterize finite Radon measures we define the positive cone $\stackrel{+}{B}_{p q}^{s}\left(\mathbb{R}^{n}\right)$ as the collection of all positive $f \in B_{p q}^{s}\left(\mathbb{R}^{n}\right)$.

Theorem 2.2. Let $M\left(\mathbb{R}^{n}\right)$ be the collection of all finite Radon measures. Then

$$
M\left(\mathbb{R}^{n}\right)=\stackrel{+}{B}_{1 \infty}^{0}\left(\mathbb{R}^{n}\right)
$$

and

$$
\mu\left(\mathbb{R}^{n}\right) \sim\left\|\mu \mid B_{1 \infty}^{0}\left(\mathbb{R}^{n}\right)\right\|, \quad \mu \in M\left(\mathbb{R}^{n}\right) .
$$

Proof. By the proof in [5, pp. 82, 83, Proposition 1.127],

$$
\left\|\mu \mid B_{1 \infty}^{0}\left(\mathbb{R}^{n}\right)\right\| \leq \mu\left(\mathbb{R}^{n}\right) \quad \text { if } \quad \mu \in M\left(\mathbb{R}^{n}\right) .
$$

In order to prove the converse inequality, one use the characterisation of Besov spaces via local means. Let $k_{0}$ be a $C^{\infty}$ non-negative function with

$$
\operatorname{supp} k_{0} \subset\{x:|x| \leq 1\} \text { and } \overline{k_{0}(0) \neq 0 .}
$$

If $f \in \stackrel{+}{B}_{1 \infty}^{0}\left(\mathbb{R}^{n}\right)$, then $f=\mu$ is a tempered measure. By [5, p. 10, Theorem 1.10],

$$
\left\|\mu\left|B_{1 \infty}^{0}\left(\mathbb{R}^{n}\right)\|\geq c\| k_{0}(1, \mu)\right| L_{1}\left(\mathbb{R}^{n}\right)\right\|=c \int_{\mathbb{R}^{n} \mathbb{R}^{n}} k_{0}(x-y) d \mu(y) d x .
$$

Applying Fubini's theorem, one gets

$$
\left\|\mu \mid B_{1 \infty}^{0}\left(\mathbb{R}^{n}\right)\right\| \geq c \mu\left(\mathbb{R}^{n}\right) .
$$


Corollary 2.3. Let $f \in L_{1}\left(\mathbb{R}^{n}\right)$ and $f(x) \geq 0$ almost everywhere. Then

$$
\left\|f\left|L_{1}\left(\mathbb{R}^{n}\right)\|\sim\| f\right| B_{1 \infty}^{0}\left(\mathbb{R}^{n}\right)\right\| .
$$

Proof. Let $\mu=f \mu_{L}$, where $\mu_{L}$ is the Lebesgue measure. Then

$$
\mu\left(\mathbb{R}^{n}\right)=\int_{\mathbb{R}^{n}} f(x) \mu_{L}(d x)=\left\|f \mid L_{1}\left(\mathbb{R}^{n}\right)\right\|
$$

and

$$
\left\|\mu\left|B_{1 \infty}^{0}\left(\mathbb{R}^{n}\right)\|=\| f\right| B_{1 \infty}^{0}\left(\mathbb{R}^{n}\right)\right\| .
$$

From (11) follows the statement in the Corollary.

The question arises whether Corollary 2.3 can be extended to all $f \in L_{1}\left(\mathbb{R}^{n}\right)$. We have

$$
L_{1}\left(\mathbb{R}^{n}\right) \hookrightarrow B_{1 \infty}^{0}\left(\mathbb{R}^{n}\right) \text {, hence }\left\|f\left|B_{1 \infty}^{0}\left(\mathbb{R}^{n}\right)\|\leq c\| f\right| L_{1}\left(\mathbb{R}^{n}\right)\right\|
$$

for all $f \in L_{1}\left(\mathbb{R}^{n}\right)$. But the converse is not true even for functions $f \in L_{1}\left(\mathbb{R}^{n}\right)$ with compact support in the unit ball.

Proposition 2.4. There are functions $f_{j} \in L_{1}\left(\mathbb{R}^{n}\right)$ with

$$
\operatorname{supp} f_{j} \subset\{y:|y| \leq 1\}, \quad j \in \mathbb{N},
$$

such that $\left\{f_{j}\right\}$ is a bounded set in $B_{1 \infty}^{0}\left(\mathbb{R}^{n}\right)$, but

$$
\left\|f_{j} \mid L_{1}\left(\mathbb{R}^{n}\right)\right\| \rightarrow \infty \quad \text { if } j \rightarrow \infty .
$$

Proof. We may assume $n=1$.

Let $a \in C^{1}(\mathbb{R})$ be an odd function with

$$
\operatorname{supp} a \subset\{x:|x| \leq 2\}, \quad a(x) \geq 0, \quad x \geq 0
$$

and

$$
\max _{-2 \leq x \leq 2}|a(x)|=|a(-1)|=a(1)=1 .
$$

If $c=\max _{-2 \leq x \leq 2}\left|a^{\prime}(x)\right|$, then $c \geq 1$. Define $a_{0} \in C^{1}(\mathbb{R})$ by

$$
a_{0}(x)=c^{-1} a(x) .
$$

Then one has for any $x \in \mathbb{R}$,

$$
\left|a_{0}(x)\right| \leq c^{-1} \leq 1, \quad\left|a_{0}^{\prime}(x)\right| \leq 1, \quad \text { and } \quad \int_{\mathbb{R}} a_{0}(x) d x=0 .
$$


Define a function $a_{\nu}, \nu \in \mathbb{N}$, by

$$
a_{\nu}(x)=2^{\nu} a_{0}\left(2^{\nu} x\right)
$$

Then

$$
\operatorname{supp} a_{\nu} \subset\left[-2^{-\nu+1}, 2^{-\nu+1}\right]
$$

and

$$
\left|a_{\nu}(x)\right| \leq c^{-1} 2^{\nu}, \quad\left|a_{\nu}^{\prime}(x)\right| \leq 2^{2 \nu}, \quad \int_{\mathbb{R}} a_{\nu}(x) d x=0 .
$$

According to [5, p. 12, Definition 1.15], $a_{0}$ is an $1_{1}$-atom and $a_{\nu}$ are $(0,1)_{1,1}$-atoms. It follows from [4, Theorem 13.8] that $\sum_{\nu=1}^{\infty} a_{\nu}(x)$ converges in $S^{\prime}\left(\mathbb{R}^{n}\right)$ and represents an element of $B_{1 \infty}^{0}\left(\mathbb{R}^{n}\right)$. Let $f \stackrel{S^{\prime}}{=} \sum_{\nu=1}^{\infty} a_{\nu}$

Let

$$
f_{j}(x)=\sum_{\nu=1}^{j} a_{\nu}(x)
$$

Then $\operatorname{supp} f_{j} \subset[-1,1]$,

$$
\begin{aligned}
\left\|f_{j} \mid L_{1}\left(\mathbb{R}^{n}\right)\right\| & \geq \int_{0}^{+\infty} f_{j}(x) d x=\int_{0}^{+\infty} \sum_{\nu=1}^{j} a_{\nu}(x) d x \\
& =j \int_{0}^{+\infty} a_{0}(x) d x \rightarrow \infty, \quad j \rightarrow \infty .
\end{aligned}
$$

On the other hand one has by the above atomic argument

$$
\left\|f_{j} \mid B_{1 \infty}^{0}(\mathbb{R})\right\| \leq 1 \quad \text { for } \quad j \in \mathbb{N} \text {. }
$$

Corollary 2.5. Not any characteristic function of a measurable subset of $\mathbb{R}^{n}$ is a pointwise multiplier in $B_{1 \infty}^{0}\left(\mathbb{R}^{n}\right)$.

Proof. Let $f \in L_{1}\left(\mathbb{R}^{n}\right)$ real. Let $M_{+}$be a set of points $x$ such that $f(x) \geq 0$ and $M_{-}=\{x: f(x)<0\}$. Then

$$
\left\|f\left|L_{1}\left(\mathbb{R}^{n}\right)\|=\| \chi_{M_{+}} f\right| L_{1}\left(\mathbb{R}^{n}\right)\right\|+\left\|\chi_{M_{-}} f \mid L_{1}\left(\mathbb{R}^{n}\right)\right\|,
$$

where $\chi_{M_{+}}, \chi_{M_{-}}$are characteristic functions of sets $M_{+}$and $M_{-}$respectively. One may apply Corollary 2.3 to the functions $\chi_{M_{+}} f$ and $\chi_{M_{-}} f$ and get

$$
\left\|f\left|L_{1}\left(\mathbb{R}^{n}\right)\|\leq c\| \chi_{M_{+}} f\right| B_{1 \infty}^{0}\left(\mathbb{R}^{n}\right)\right\|+c\left\|\chi_{M_{-}} f \mid B_{1 \infty}^{0}\left(\mathbb{R}^{n}\right)\right\| .
$$


If any characteristic function of a set in $\mathbb{R}^{n}$ would be a pointwise multiplier in $B_{1 \infty}^{0}\left(\mathbb{R}^{n}\right)$, then

$$
\left\|\chi_{M_{+}} f\left|B_{1 \infty}^{0}\left(\mathbb{R}^{n}\right)\|\leq c\| f\right| B_{1 \infty}^{0}\left(\mathbb{R}^{n}\right)\right\|, \quad\left\|\chi_{M_{-}} f\left|B_{1 \infty}^{0}\left(\mathbb{R}^{n}\right)\|\leq c\| f\right| B_{1 \infty}^{0}\left(\mathbb{R}^{n}\right)\right\|,
$$

hence

$$
\left\|f\left|L_{1}\left(\mathbb{R}^{n}\right)\|\leq c\| f\right| B_{1 \infty}^{0}\left(\mathbb{R}^{n}\right)\right\| .
$$

Since for any function $f \in L_{1}\left(\mathbb{R}^{n}\right)$ holds

$$
\left\|f\left|B_{1 \infty}^{0}\left(\mathbb{R}^{n}\right)\|\leq c\| f\right| L_{1}\left(\mathbb{R}^{n}\right)\right\|,
$$

one gets

$$
\left\|f\left|L_{1}\left(\mathbb{R}^{n}\right)\|\sim\| f\right| B_{1 \infty}^{0}\left(\mathbb{R}^{n}\right)\right\|, \quad \text { for real } f \in L_{1}\left(\mathbb{R}^{n}\right) .
$$

This can be also extended to complex functions $f \in L_{1}\left(\mathbb{R}^{n}\right)$. But acoording to the Proposition 2.4 this is not true.

\section{References}

[1] D. E. Edmunds and H. Triebel, Function spaces, entropy numbers, differential operators, Cambridge Tracts in Mathematics, vol. 120, Cambridge University Press, Cambridge, 1996.

[2] P. Malliavin, Integration and probability, Graduate Texts in Mathematics, vol. 157, SpringerVerlag, New York, 1995.

[3] H. Triebel, Theory of function spaces, Monographs in Mathematics, vol. 78, Birkhäuser Verlag, Basel, 1983.

[4] _ Fractals and spectra, Monographs in Mathematics, vol. 91, Birkhäuser Verlag, Basel, 1997.

[5] _ Theory of function spaces, III, Monographs in Mathematics, vol. 100, Birkhäuser Verlag, Basel, 2006. 